\title{
Commercial fish ELISA kits have a limited capacity to detect different fish species and their products
}

Short title: Limited capacity of detecting fish utilizing ELISA technology

Authorship: Thimo Ruethers ${ }^{a, b, c, d}$, Aya C. Taki ${ }^{a, b, c, d}$, Jasmit Khangurha ${ }^{e}$, James Roberts $^{\mathrm{e}}$, Saman Buddhadasa ${ }^{\mathrm{e}}$, Dean Clarke $^{\mathrm{e}}$, Claire E. Hedges ${ }^{\mathrm{e}}$, Dianne E. Campbell ${ }^{b, f, g}$, Sandip D. Kamatha, ${ }^{a, b, c, d}$, Andreas L. Lopata ${ }^{a, b, c, c d}$, Martina Koeberle† $^{\dagger}$

\section{Authors' Affiliation}

aMolecular Allergy Research Laboratory, College of Public Health, Medical and Veterinary Sciences, James Cook University, 1 James Cook Dr, Douglas, Queensland 4814, Australia;

${ }^{b}$ Centre for Food and Allergy Research, Murdoch Children's Research Institute, Flemington Rd, Parkville, Victoria 3052, Australia;

cAustralian Institute of Tropical Health and Medicine, James Cook University, 1 James Cook Dr, Douglas, Queensland 4814, Australia;

${ }^{\mathrm{d} C e n t r e}$ for Sustainable Tropical Fisheries and Aquaculture, Faculty of Science and Engineering, James Cook University, 1 James Cook Dr, Douglas, Queensland 4814, Australia;

eNational Measurement Institute, 1/153 Bertie Street, Port Melbourne, Victoria 3207, Australia

fChildren's Hospital at Westmead, Allergy and Immunology, Locked Bag, Westmead, New South Wales 2145, Australia;

This is the author manuscript accepted for publication and has undergone full peer review but has not been through the copyediting, typesetting, pagination and proofreading process, which may lead to differences between this version and the Version of Record. Please cite this article as doi: $10.1002 /$ jsfa.10451

This article is protected by copyright. All rights reserved. 
gDiscipline of Paediatrics and Child Health, University of Sydney, Fisher Road, Sydney, New South Wales 2006, Australia

\section{Corresponding Author Contact Details}

${ }^{\dagger}$ Martina Koeberl, PhD

martina.koeberl@measurement.gov.au

Allergen Research Scientist, Analytical Service Branch,

National Measurement Institute,

1/153 Bertie Street, Port Melbourne, Victoria 3207, Australia

Email: martina.koeberl@my.jcu.edu.au

Phone: +61 396444888

\section{Authors' e-mail Addresses}

thimo.ruethers@my.jcy.edu.au; aya.taki@unimelb.edu.au;

jasmit.khangurha@measurement.gov.au; james.roberts@measurement.gov.au;

saman.buddhadasa@measurement.gov.au; dean.clarke@measurement.gov.au;

claire.hedges@measurement.gov.au; dianne.campbell1@health.nsw.gov.au;

sandip.kamath@jcu.edu.au; andreas.lopata@jcu.edu.au;

martina.koeberl@my.jcu.edu.au

This article is protected by copyright. All rights reserved. 


\section{Abstract}

BACKGROUND: Fish is a major food and allergen source, requiring declaration on packaged food, often ensured by employing ELISAs. Over 1,000 different fish species are traded and consumed worldwide, increasingly provided by aquaculture. Up to $3 \%$ of the general population are at risk of sometimes fatal allergic reactions to fish, requiring strict avoidance of this food commodity. The aim of this study is to evaluate the capacity of three commercially available ELISA tests to detect a wide variety of bony and cartilaginous fish and their products, essential to ensure reliable and safe food labeling.

RESULTS: The detection of 57 bony fish ranged from $26 \%$ to $61 \%$. Common European and North American species including carp, cod, and salmon species demonstrated a higher detection rate as compared to those from the Asia-Pacific, including pangasius and several mackerel and tuna species. Among the 17 canned bony fish products, only $65 \%$ to $86 \%$ were detected, with tuna showing the lowest rate. None of the cartilaginous fish $(n=9)$ as well as other vertebrates $(n=8)$ or shellfish $(n=5)$ were detected.

CONCLUSIONS: We demonstrate a limited capacity of three commercial fish ELISA kits to detect fish and their products. The complexity of fish as an increasing utilized protein source raises the urgent need for improved detection methods, crucial for the food industry to provide safe seafood products and comply with international legislations. 
Keywords: Bony fish; cartilaginous fish; fish allergy; fish cross-reactivity; food labeling; food safety. 


\section{INTRODUCTION}

Adverse reactions to food can be fatal and include metabolic, toxic and allergic reactions. ${ }^{1}$ Food allergy is an increasing health burden worldwide,${ }^{2}$ with fish being among the top eight food groups causing $90 \%$ of allergic reactions in consumers. ${ }^{3-5}$ Fish allergy is typically a life-long disease, ${ }^{6}$ with sensitization rates of up to $3 \%$ in the general population. ${ }^{7}$ In the Asia-Pacific region, fish is a staple protein source and one of the most common triggers of allergic anaphylaxis. ${ }^{8}$ Among the over 34,000 described fish species, a minimum of over 1,000 are consumed globally. Unlike comprehensively studied food allergen sources, including egg, peanut and milk, the allergen content and variety of allergenic proteins differs between fish species. ${ }^{9-11}$ Currently available diagnostic and detection tests do not address the complexity of species-specific sensitisation patterns. In addition, species-specific recommendations often cannot be implemented due to mislabeling and ambiguous fish names. ${ }^{12-14}$ The management of fish allergy is therefore currently limited to strict avoidance of all fish and fish-containing products. ${ }^{15}$ Consequently, affected consumers rely on safe and correct food labeling. This is of particular importance considering the fast growth of the aquaculture industry and the increasing consumption of seafood, including fish, worldwide. ${ }^{16}$ In all countries implementing mandatory allergen labeling (currently over 60 ), fish needs to be declared on packaged food, with the exception of Japan and Taiwan. ${ }^{17}$ Therefore, the reliable detection and quantification of fish in processed food is essential, not only for the health and safety of consumers, but also to meet regulatory requirements. 
Analytical food laboratories worldwide test products to ensure correct food labeling; however, false positive and negative test results can occur. ${ }^{18,19}$ False negative detections could be potentially fatal for consumers with a respective allergy. In contrast, false positive results can lead to expensive, unwarranted recalls of food products, reduced food choices, increased food costs and a loss of trust and reputation of the food allergen testing community. Food recalls cause an estimated average global annual direct cost of US\$10m to the food industry. In the United States of America, 33\% of food recalls between 2009 and 2019 have been due to undeclared allergens; $8 \%$ of those have been due to undeclared fish (United States Department of Agriculture - Food Safety and Inspection Services, https://www.fsis.usda.gov, accessed 31 January 2020). In comparison, this has been $31 \%$ and $4 \%$ in the European Union in the same timeframe (European Commission - Food Safety - The Rapid Alert System for Food and Feed; https://ec.europa.eu/food/safety/rasff_en, accessed 31 January 2020).

Methods for the detection and quantification of food allergen sources include Enzymelinked Immunosorbent Assays (ELISA), lateral flow devices (LFDs), polymerase chain reaction $(\mathrm{PCR}),{ }^{18,20-23}$ capillary zone electrophoresis, ${ }^{24}$ superparamagnetic nanoparticle-based lateral flow immunoassay, ${ }^{25}$ liquid chromatography coupled with mass spectrometry (LC/MS), ${ }^{17,26}$ and multiplex systems. ${ }^{27,28}$ However, all of the available methods have certain advantages and disadvantages, often leading to a limited utility for the food industry and regulatory institutions. ${ }^{29-32} \mathrm{~A}$ recent evaluation of commercial peanut ELISA kits, for example, demonstrated that the sensitivity and 
selectivity vary greatly, mostly due to different protein targets. ${ }^{33}$ Azarnia et al. demonstrated that both ELISA and LC/MS failed to detect egg in cooked pasta. ${ }^{34}$ Most of the methods for fish quantification have been developed for the identification of species or detection of the major fish allergen parvalbumin. ${ }^{5,35}$ Nevertheless, the only methods commercially available for the detection and quantification of fish are ELISA, LFDs and PCR.

Commercially available DNA-based methods for the detection of fish (and other vertebrate species) are available from several manufacturers including Agilent (USA), Minerva Biolabs (Germany), R-Biopharm (Germany), and Techne (UK). However, all kits are designed to detect specific fish species or a narrow range of species only. At the time of this study, there were only two manufacturers of commercial fish ELISA kits known to us, namely Romer Labs (Austria, UK) and XEMA (Russia). These ELISA kits have been developed to detect primarily bony fish from Europe and North America, utilizing antibodies raised against cod, presumably Atlantic cod (Gadus morhua). This species is found exclusively in Europe and North America and differs greatly from most species consumed in and exported from other regions, including the AsiaPacific. ${ }^{9,11,36,37}$ This region contributes $89 \%$ of the global aquaculture and over $50 \%$ of the world's wild fish catch (Asia-Pacific Fishery Commission, FAO 2019). Some inhouse ELISA assays have been developed for different fish species, ${ }^{38-40}$ however, they are not commercially available. It is to note that none of these ELISA approaches are suitable for fish species authentication. 
Fish as a food supply is very complex with over 1,000 different fish species harvested and consumed globally, making its detection challenging. For accurate and safe food labeling it is essential to have tools for the detection of contaminations and samples originating from any bony fish (Actinopterygii) as well as cartilaginous fish (Chondrichthyes) including sharks and rays. Figure S1 illustrates the taxonomic relationship of fish, non-fish vertebrates (Tetrapoda), and shellfish (Mollusca and Arthropoda) and demonstrates the close relation of both classes of fish. Subsequently the detection of proteins from non-fish vertebrates or shellfish would imply a false positive result.

This study aimed to evaluate three commercially available fish ELISA kits for their capacity to detect a wide variety of bony and cartilaginous fish species consumed worldwide. 


\section{MATERIAL AND METHODS}

\section{Extract preparation}

Details about species and corresponding taxonomic classification of the fish and other animals analyzed in this study are provided in Table 1. Whole specimen, where available, or fillets from commonly consumed 57 bony and nine cartilaginous fish were sourced from fishermen, retailers and fellow researchers. Muscle tissue samples from the center of the fillets were taken and stored at $-80^{\circ} \mathrm{C}$ until further use. Samples from other vertebrates and shellfish were sourced accordingly. Canned fish products were purchased and the brine drained prior to analyses (see Table S1 for product details). For protein extraction, tissue samples were thawed on ice and homogenized with a rotor-stator homogenizer (5 minutes at 13,000 rpm on ice) in phosphate-buffered saline (PBS, $10 \mathrm{mM}$ phosphate; $\mathrm{pH} 7.2 ; 2 \mathrm{ml} / \mathrm{g}$ tissue). After gentle agitation overnight at $4^{\circ} \mathrm{C}$, subsequent centrifugation $(20,000 \times \mathrm{g})$ and filtration, extracts were stored at $80^{\circ} \mathrm{C}$ until further use, referred to as raw extracts. For the heated extracts, tissue was heated in PBS $\left(95-100^{\circ} \mathrm{C}\right)$ for 20 minutes before homogenization and extraction as detailed above.

\section{Fish ELISA kits}

Three commercially available ELISA kits were used in this study: AgraQuant Fish ELISA kit from Romer Labs (UK, Austria) as well as Common Bone Fish Antigen EIA ELISA kit, versions 2 and 3, from XEMA (Russia). Both manufacturers provided their ELISA kits free of charge for this study. According to the manufactures, the AgraQuant 
Fish ELISA is detecting parvalbumin from cod, whereas the Common Bone Fish Antigen ELISA kit is detecting proteins of the muscular tropomyosin complex from cod. Both proteins are known to be heat-stable ${ }^{5}$ and the manufacturers instructions for all three kits states their suitability to detect fish in raw and heat-treated products.

The protein extracts were thawed, vortexed and centrifuged for 5 minutes at $17,000 \times \mathrm{g}$. The supernatants were used for subsequent testing, performed according to the manufacturers' protocols and instructions. Atlantic cod (Gadus morhua), presumably the cod species the antibodies used in the kit were raised against, was used as reference fish species throughout all analyses. The raw and heated extracts from Atlantic cod were diluted to result in absorbance values between the two highest standards of the respective ELISA kits (Figure 1). With the exception of the canned fish products, all extracts were diluted and tested accordingly, correlating to the dilution for the respective Atlantic cod extract. The raw and heated cod extract was used as positive control for each ELISA kit batch, while the buffer was used as negative control. All samples were analyzed using duplicate wells of the ELISA kit. All samples were analyzed once using duplicate wells, representing standard procedures for most analytical food laboratories. Therefore, no comprehensive statistical analysis can be performed for the data generated in this study. Results are referred to ELISA kit 1, ELISA kit 2, and ELISA kit 3, not disclosing their correspondence to the utilized commercial kits. The optical density (OD) of individual samples and ELISA kits standards were measured at the wavelength specified by the manufacturer, using a microtiter plate reader (Infinite 200 Pro (Tecan, Australia)). The obtained OD values 
for all samples were divided by the OD value for standard 1 of the respective ELISA kit, representing the limit of quantification (LOQ). The obtained ratio of this division (referred to as "fold increase" in the remaining article) was plotted as bar graphs in the figures. The fold increase of 1 equals the LOQ for the test kits, and a fold increase of 0.5 equals half of the LOQ ( $1 / 2$ LOQ), which will also be referred as limit of detection (LOD) as published previously. ${ }^{41}$ The area between the calculated fold increases of 0.5-1 is shaded in grey. These results are considered inconclusive, being neither 'detected' nor 'not detected', in line with procedures on reporting results in most analytical food laboratories.

\section{SDS-PAGE and immunoblotting}

The raw and heated extracts from Atlantic cod (Gadus morhua) were evaluated for their protein distribution by SDS-PAGE. The presence of parvalbumin and tropomyosin was confirmed by immunoblotting and mass spectrometry as described previously. ${ }^{42}$ 


\section{RESULTS AND DISCUSSION}

Food testing laboratories worldwide depend on commercially available tools for the detection of allergenic food sources crucial for the food industry to provide safe products and comply with international legislations. Food allergens can be quantified using different analytical methods, however ELISA based systems are most frequently employed. The quantification capacity of commercial ELISAs cannot directly be compared as the manufacturers generate their own antibodies, conjugates, standards and calibrants used in their respective kits.

\section{Comparison of ELISA kits}

The ELISA kits utilized in this study were evaluated for their capacity to detect a wide range of fish species. The three ELISA kits differ from each other in the detection range, LOD, LOQ, sample extraction buffer ratio, calibrants and units expressed, making a quantitative comparison not possible. However, according to the manufacturers, all three ELISA systems use cod as their reference fish species, which the authors hypothesize is Atlantic cod (Gadus morhua). The AgraQuant Fish ELISA kit uses antibodies against fish proteins, parvalbumin, and results are expressed in ppm cod, according to the manufacturer's instructions. The Common Bone Fish Antigen EIA ELISA kit versions 2 and 3 utilize monoclonal antibodies against a protein of the muscular tropomyosin complex and results are expressed in units $/ \mathrm{ml}$. Both antibody targets, parvalbumin and tropomyosin, are heat-stable and commonly found in muscle tissue of fish. Additional details of the target and antibody are not provided 
by the manufacturers or distributors. Raw and heated cod extracts were evaluated for their distribution of proteins by SDS-PAGE (see Figure S2), and used as reference and positive control throughout this study. The presence of parvalbumin and tropomyosin in both extracts was demonstrated by immunoblotting. Using a parvalbumin-specific antibody the signal was similar for both raw and heated extracts, while the signal using a tropomyosin-specific antibody was much stronger for the heated as compared to raw extract. Parvalbumin is the major fish allergen, while tropomyosin, a well-known pan-allergen in arthropods and mollusks, has recently also been described as a fish allergen for a few fish species. ${ }^{5,42}$ However, these two tropomyosin proteins share only a very low amino acid homology with $51-58 \% .^{5}$ Tropomyosin is a structural protein and plays a critical role in regulating actin filaments and is most abundant in muscle tissue. Parvalbumin is a calcium-binding protein, important for muscle contraction, and its abundance can differ within one specimen. ${ }^{43,44}$ To exclude this factor of variance, tissue samples were taken from the middle of the fillets, the most frequently consumed part of fish.

\section{Test procedure establishment for extract dilution}

The extract preparation was standardized by using the same amount of fish tissue for the protein extraction. In-house prepared raw and heated extracts from Atlantic cod were used as reference in all analyses. The extracts were diluted following the ELISA kit extraction protocol for each ELISA kit individually, to fall within the standard range of the respective ELISA kit. The standard curve of the respective ELISA kit, as 
provided with the quality certificate of the ELISA kit lot number, is shown in Figure 1. The triangles represent the values for the cod extracts located between the two highest standards. The canned fish products were tested undiluted as a low intact protein content due to extreme food processing was expected. All other raw and heated samples were diluted according to the respective extract from cod. The dilution factors varied for raw and heated samples and the three different ELISA kits. The AgraQuant Fish ELISA kit was more sensitive to the cod raw extract (approximately 2.5 times more sensitive). In contrast, the Common Bone Fish Antigen EIA ELISA kits (Versions 2 and 3 (XEMA, Russia)) detected lower concentrations of heated cod extract (approximately 2.3 times more sensitive). Both parvalbumin and tropomyosin are heatstable proteins and present in the raw as well as heated positive control (Figure S2). However, the relative abundance of tropomyosin seems much lower in the raw as compared to the heated extract.

\section{Detection of fish}

For this study, results between LOD and LOQ were considered inconclusive and not included as detections. In a routine laboratory setting these results are commonly considered as either detected or not detected depending on internal procedures. All samples were prepared using the same dilution factor and without food matrix. A higher dilution factor and/or testing with food matrix would lower the rate of positive test results even more. Negative test results can therefore be rendered as nondetection of the fish species in food products. 


\section{Detection of bony fish species}

The results of 57 analyzed fish species, represented as fold-increase of LOQ for each ELISA kit (kit 1-3 in A-C, respectively) are shown in Figure 2. At least one of the two extracts, raw and heated, from $93 \%$ of the analyzed species was detected by kit 1 ; this was $67 \%$ for kit 2, and $81 \%$ for kit 3 . For the 57 raw extracts, kit 1 detected $86 \%$, kit 2 $65 \%$, and kit $375 \%$. For heated extracts, the detection rate was lower with $47 \%$ for kit 1, $63 \%$ for kit 2, and 32\% for kit 3 . Kit 1 detected both raw and heated extracts from $40 \%$ of the analyzed species; this was $61 \%$ for kit 2 and $26 \%$ for kit 3 .

Only $21 \%$ of species, including cod, carp, and rainbow trout, were detected by all three ELISA kits. Eight of those are the only analyzed representatives of the fish orders Beryciformes, Cypriniformes, Gadiformes, and Osmeriformes, including some of the most commonly consumed species in Europe and North America, i.e. Atlantic cod, European carp. The other four species belong to the Perciformes, the largest of all fish orders as well as Salmoniformes. Both raw and heated extracts from six species commonly consumed in Europe and North America (Atlantic cod, Atlantic salmon, European carp, rainbow trout, European pilchard, and orange roughy) were detected by all three ELISA kits, except heated extracts from Atlantic salmon and pilchard, which were only detected by kit 1 and 2 , respectively.

Raw and heated extracts from king threadfin and frypan bream/snapper, both only found in the Asia-Pacific region, were 'not detected' by any of the three ELISA kits. Furthermore, kit 1 and kit 3 did not detect extracts from Asian swamp eel and pink 
snapper. Additionally, kit 2 and 3 did not detect extracts from goldband snapper. Kit 2 also did not detect extracts from ling, mahi mahi, crimson snapper, parrot fish, six tuna and mackerel species, two coralgrouper, swordfish, ocean jacket, and John Dory. Moreover, kit 3 did not detect any extracts from the four emperor species. Most species 'not detected' were from fish originating from the Asia-Pacific region and are traded worldwide. It is to note that king threadfin and frypan bream/snapper are the only two bony fish from which neither the raw nor the heated extract were detected by any of the three kits. Kit 2 demonstrated the highest rate of detecting both raw and heated fish extracts (61\%) from 57 bony fish species and was the only kit which detected both raw and heated extracts from Atlantic salmon, barramundi, pangasius, and tilapia; four of the most frequently consumed species and common trigger of allergic reactions in the Asia-Pacific region. ${ }^{45}$ However, this kit also demonstrated the lowest overall detection rate with $67 \%$ neither raw nor heated extracts from 19 fish species.

All analyzed extracts demonstrated lower reactivity compared to Atlantic cod extract, except three raw and two heated extracts with kit 1, four raw and four heated with kit 2, and three raw and three heated with kit 3. Eight of 19 extracts with high reactivity derived from Pacific cod and Southern blue whiting, both belonging to the same family as Atlantic cod (Gadidae).

Using kit 1, 2, 3, resulted in inconclusive/'not detected' results for 10\%, 14\%, 15\% respectively of all raw extracts $(n=57)$ and $26 \%, 17 \%, 19 \%$ respectively of all heated extracts $(n=57)$, due to an inconclusive low value between LOD and LOQ. Further 
analysis would be required to determine if these results can be considered as detection, including increasing the concentration of the sample extract.

\section{Detection of canned bony fish samples}

In addition to detecting raw and heated fish extracts, the capacity of the ELISA kits to detect fish after extensive processing was also assessed. Canned fish products ( $n=17)$ were analyzed undiluted as the value for most heated extracts from corresponding fish species were below LOQ (see 3.2.2). Two out of three ELISA kits did not detect heated extracts from Atlantic salmon and European pilchard, while all heated tuna extracts were below LOQ with all kits (Table 1).

In contrast, all canned salmon $(n=8)$ and one canned pilchard/sardine $(n=2)$ were detected by all three ELISA kits (Figure 3 and Table S1). The other canned pilchard/sardine sample (sardine 1 ) was detected only by kit 2 . Kit 2 detected only one canned tuna sample ( $n=7)$, while kit 1 and 3 detected four ( $n=7)$. One tuna (tuna 5) sample was not detected by any of the kits and no sample detection pattern was found. Values for canned salmon were in average 4.5-fold of LOQ and for canned tuna 1.0fold. In average, the values were the highest with kit 3 (6.4 and 1.2, respectively) followed by kit 1 (4.6 and 1.2, respectively) and kit 2 (2.4 and 0.6, respectively). For all investigated kits three different canned tuna preparations obtained values between the LOQ and LOD and considered inconclusive. Further testing could potentially result in detection of these samples. However, in contrast to raw and heated sample extracts the concentration of canned samples cannot be increased since they are already 
undiluted. Considering the detection capacity for canned fish, an inability to detect fish in a mixed food sample, containing canned or processed fish, is to be expected. This is particularly anticipated when fish content originates from tuna or pilchard/sardine.

\section{Detection of cartilaginous fish species}

The group of fish is subdivided into bony and cartilaginous fish (Figure S1), the latter is often sold as fillet or in processed food and includes shark, ray, skate, flake, dogfish, spurdog, or Schillerlocke. A lower allergenicity was recently suggested for cartilaginous fish. ${ }^{46}$ However, the possible low allergenicity is region- and patientspecific,,$^{5,47-49}$ and fish-allergic patients should, therefore, avoid all fish including cartilaginous fish.

In this study, raw and heated extracts from nine cartilaginous fish were analyzed, representing a wide variety of species consumed in the Asia-Pacific and Europe (Table 1). The generated values were below LOQ for all 18 samples with all three ELISA kits. Values for heated extracts from longsnout dogfish and shortnose spurdog were inconclusive for kit 2, and for all other extracts below the LOD for all three ELISA kits (Figure 4).

The demonstrated inability of the ELISA kits to detect cartilaginous fish is a major drawback and poses a serious risk to fish-allergic consumers, particular important in the Asia-Pacific region where consumption of cartilaginous fish is high. ${ }^{50-52}$ It is to note that the manufacturer XEMA states that the two ELISA kits are specific for bony fish 
only and an immunoreactivity of $<1 \%$ for sharks, as compared to cod, is to be expected according to the manufacturers' information.

\section{Detection of non-fish vertebrates and shellfish}

The risk of false positive results was evaluated by analyzing samples from non-fish vertebrates $(n=8)$ including mammals, birds, amphibians and reptiles as well as shellfish $(n=5)$ including mollusks and crustaceans (Table 1). None of these extracts were detected, suggesting a low probability of false positive results with any of the three evaluated ELISA kits (Figure 4). However, heated turtle (kit 2) and raw squid extracts (kit 1) showed a value greater than 0.5 of the LOQ.

\section{CONCLUSIONS}

All three investigated ELISA kits demonstrated a limited capacity to detect both raw and heated extracts from bony fish $(26-61 \% ; n=57)$, canned bony fish $(65-86 \% ; n=17)$ as well as cartilaginous fish $(0 \% ; n=9)$. Importantly a significant number of species frequently consumed globally had a low detection rate. Fish is one of the most diverse food groups and frequently triggers severe allergic reactions. This diversity requires consideration in the development of urgently needed improved detection methods. Only improved methods can ensure safe and correct food labeling, required to meet regulatory obligations.

This article is protected by copyright. All rights reserved. 


\section{ACKNOWLEDGMENTS}

The authors thank Denis Erceg from BioSys (BioSys Australia Pty Ltd, Australia) for the communication and help with, and both XEMA (XEMA Co., Ltd. of Moscow, Russia) and BioSys for providing complimentary XEMA ELISA kits. Chiara Palladino and Adrian Rogers from Romer Labs (Romer Labs, Austria and Romer Labs, UK) for complimentary ELISA kits. We would like to acknowledge Wytamma Wirth from the Centre for Tropical Environmental and Sustainability Science as well as Roni Nugraha and Shaymaviswanathan Karnaneedi from the Molecular Research Laboratory (James Cook University, Townsville, Australia) and Berit Bang (The Arctic University of Norway, Tromsø, Norway) for providing tissue samples.

TR is a PhD full-time scholar of the Centre for Food and Allergy Research, Australia. SK is a National Health and Medical Research Council (NHMRC, Australia) Peter Doherty Early Career Research Fellow [GNT1124143]. The study was financially supported by an NHMRC grant to AL and DC [APP1086656].

\section{Conflict of Interest}

The authors declare no conflict of interest. 


\section{REFERENCES}

1. Renz H, Allen KJ, Sicherer SH, Sampson HA, Lack G, Beyer K and Oettgen HC, Food allergy. Nat Rev Dis Primers 4:17098 (2018).

2. Prescott $\mathrm{S}$ and Allen $\mathrm{KJ}$, Food allergy: riding the second wave of the allergy epidemic. Pediatr Allergy Immunol 22:155-160 (2011).

3. Kuehn A, Swoboda I, Arumugam K, Hilger C and Hentges F, Fish allergens at a glance: variable allergenicity of parvalbumins, the major fish allergens. Front Immunol 5:179 (2014).

4. Lopata AL and Lehrer SB, New insights into seafood allergy. Curr Opin Allergy Clin Immunol 9:270-277 (2009).

5. Ruethers T, Taki AC, Johnston EB, Nugraha R, Le TTK, Kalic T, McLean TR, Kamath SD and Lopata AL, Seafood allergy: A comprehensive review of fish and shellfish allergens. Mol Immunol 100:28-57 (2018).

6. Sharp MF and Lopata AL, Fish allergy: in review. Clin Rev Allergy Immunol 46:258-271 (2014).

7. Moonesinghe $\mathrm{H}$, Mackenzie $\mathrm{H}$, Venter $\mathrm{C}$, Kilburn S, Turner $\mathrm{P}$, Weir $\mathrm{K}$ and Dean $\mathrm{T}$, Prevalence of fish and shellfish allergy: A systematic review. Ann Allergy Asthma Immunol 117:264-272 e264 (2016).

8. Le TTK, Nguyen DH, Vu ATL, Ruethers T, Taki AC and Lopata AL, A cross-sectional, populationbased study on the prevalence of food allergies among children in two different socio-economic regions of Vietnam. Pediatr Allergy Immunol 30:348-355 (2019).

9. Ruethers T, Raith M, Sharp MF, Koeberl M, Stephen JN, Nugraha R, Le TTK, Quirce S, Nguyen HXM, Kamath SD, Mehr SS, Campbell DE, Bridges CR, Taki AC, Swoboda I and Lopata AL, Characterization of Ras $\mathrm{k} 1$ a novel major allergen in Indian mackerel and identification of parvalbumin as the major fish allergen in 33 Asia-Pacific fish species. Clin Exp Allergy 48:452-463 (2018).

10. Lee PW, Nordlee JA, Koppelman SJ, Baumert JL and Taylor SL, Evaluation and Comparison of the Species-Specificity of 3 Anti-Parvalbumin IgG Antibodies. J Agric Food Chem 59:12309-12316 (2011).

11. Liang J, Tan CC, Taylor SL, Baumert JL, Lopata AL and Lee NA, Quantitative analysis of species specificity of two anti-parvalbumin antibodies for detecting southern hemisphere fish species demonstrating strong phylogenetic association. Food Chem 237:588-596 (2017).

12. Triantafyllidis A, Karaiskou N, Perez J, Martinez JL, Roca A, Lopez B and Garcia-Vazquez E, Fish allergy risk derived from ambiguous vernacular fish names: Forensic DNA-based detection in Greek markets. Food Res Int 43:2214-2216 (2010).

13. Cawthorn D-M, Steinman HA and Witthuhn RC, DNA barcoding reveals a high incidence of fish species misrepresentation and substitution on the South African market. Food Res Int 46:30-40 (2012).

14. Huang Y-R, Yin M-C, Hsieh Y-L, Yeh Y-H, Yang Y-C, Chung Y-L and Hsieh C-HE, Authentication of consumer fraud in Taiwanese fish products by molecular trace evidence and forensically informative nucleotide sequencing. Food Res Int 55:294-302 (2014).

15. Davis CM, Gupta RS, Aktas ON, Diaz V, Kamath SD and Lopata AL, Clinical management of seafood allergy. The Journal of Allergy and Clinical Immunology: In Practice 8:37-44 (2020).

16. FAO, The State of World Fisheries and Aquaculture 2018-Meeting the sustainable development goals, Ed. FAO Rome, Italy (2018).

17. Koeberl M, Clarke D and Lopata AL, Next generation of food allergen quantification using mass spectrometric systems. J Proteome Res 13:3499-3509 (2014).

This article is protected by copyright. All rights reserved. 
18. Herrero B, Vieites JM and Espineira M, Development of an in-house fast real-time PCR method for detection of fish allergen in foods and comparison with a commercial kit. Food Chem 151:415-420 (2014).

19. Koeberl M, Clarke D, Allen KJ, Fleming F, Katzer L, Lee NA, Lopata AL, Said M, Scheelings $P$, Shepherd N, Sherlock R and Roberts J, Food Allergen Management in Australia. J AOAC Int 101:60-69 (2018).

20. Fernandes TJR, Costa J, Oliveira M and Mafra I, Exploiting 16S rRNA gene for the detection and quantification of fish as a potential allergenic food: A comparison of two real-time PCR approaches. Food Chem 245:1034-1041 (2018).

21. Hildebrandt $\mathrm{S}$, Multiplexed identification of different fish species by detection of parvalbumin, a common fish allergen gene: a DNA application of multi-analyte profiling (xMAP) technology. Anal Bioanal Chem 397:1787-1796 (2010).

22. Tetzlaff $C$ and Made D, Development of a real-time PCR system for the detection of the potential allergen fish in food. Eur Food Res Technol 243:849-857 (2017).

23. Eischeid AC, A method to detect allergenic fish, specifically cod and pollock, using quantitative real-time PCR and COI DNA barcoding sequences. J Sci Food Agric 99:2641-2645 (2019).

24. Fu L, Zhou J, Wang C, Zhang Y, Ma A and Wang Y, Determination of a major allergen in fish samples by simple and effective label-free capillary electrophoretic analysis after background suppression in ion-exchange chromatography. Food Chem 261:124-130 (2018).

25. Zheng C, Wang XC, Lu Y and Liu Y, Rapid detection of fish major allergen parvalbumin using superparamagnetic nanoparticle-based lateral flow immunoassay. Food Control 26:446-452 (2012).

26. Carrera M, Canas B and Gallardo JM, Rapid direct detection of the major fish allergen, parvalbumin, by selected MS/MS ion monitoring mass spectrometry. J Proteomics 75:3211-3220 (2012).

27. Wang W, Li Y, Zhao F, Chen Y and Ge Y, Optical thin-film biochips for multiplex detection of eight allergens in food. Food Res Int 44:3229-3234 (2011).

28. Cho CY, Nowatzke W, Oliver K and Garber EA, Multiplex detection of food allergens and gluten. Anal Bioanal Chem 407:4195-4206 (2015).

29. Goodwin PR, Food allergen detection methods: a coordinated approach. J AOAC Int 87:13831390 (2004).

30. Monaci $L$ and Visconti A, Immunochemical and DNA-based methods in food allergen analysis and quality assurance perspectives. Trends Food Sci Tech 21:272-283 (2010).

31. Poms RE, Klein CL and Anklam E, Methods for allergen analysis in food: a review. Food Addit Contam 21:1-31 (2004).

32. Senyuva $\mathrm{HZ}$, Jones IB, Sykes $M$ and Baumgartner $S, A$ critical review of the specifications and performance of antibody and DNA-based methods for detection and quantification of allergens in foods. Food Addit Contam Part A Chem Anal Control Expo Risk Assess 36:507-547 (2019).

33. Jayasena S, Smits M, Fiechter D, de Jong A, Nordlee J, Baumert J, Taylor SL, Pieters RH and Koppelman SJ, Comparison of six commercial ELISA kits for their specificity and sensitivity in detecting different major peanut allergens. J Agric Food Chem 63:1849-1855 (2015).

34. Azarnia S, Boye Jl, Mongeon V and Sabik H, Detection of ovalbumin in egg white, whole egg and incurred pasta using LC-ESI-MS/MS and ELISA. Food Res Int 52:526-534 (2013).

35. Carrera M, Cañas B and Gallardo JM, Proteomics for the assessment of quality and safety of fishery products. Food Res Int 54:972-979 (2013). 
36. Saptarshi SR, Sharp MF, Kamath SD and Lopata AL, Antibody reactivity to the major fish allergen parvalbumin is determined by isoforms and impact of thermal processing. Food Chem 148:321-328 (2014).

37. Sharp MF, Stephen JN, Kraft L, Weiss T, Kamath SD and Lopata AL, Immunological crossreactivity between four distant parvalbumins-Impact on allergen detection and diagnostics. $\mathrm{Mol}$ Immunol 63:437-448 (2015).

38. Cai QF, Wang XC, Liu GM, Zhang L, Ruan MM, Liu Y and Cao MJ, Development of a monoclonal antibody-based competitive enzyme linked-immunosorbent assay (c-ELISA) for quantification of silver carp parvalbumin. Food Control 29:241-247 (2013).

39. Chen YT and Hsieh YHP, A sandwich ELISA for the detection of fish and fish products. Food Control 40:265-273 (2014).

40. Faeste CK and Plassen C, Quantitative sandwich ELISA for the determination of fish in foods. J Immunol Methods 329:45-55 (2008).

41. Koeberl M, Sharp MF, Tian R, Buddhadasa S, Clarke D and Roberts J, Lupine allergen detecting capability and cross-reactivity of related legumes by ELISA. Food Chem 256:105-112 (2018).

42. Ruethers T, Taki AC, Nugraha R, Cao TT, Koeberl M, Kamath SD, Williamson NA, O'Callaghan $\mathrm{S}$, Nie S, Mehr SS, Campbell DE and Lopata AL, Variability of allergens in commercial fish extracts for skin prick testing. Allergy 74:1352-1363 (2019).

43. Kobayashi Y, Yang T, Yu CT, Ume C, Kubota H, Shimakura K, Shiomi K and Hamada-Sato N, Quantification of major allergen parvalbumin in 22 species of fish by SDS-PAGE. Food Chem 194:345353 (2016).

44. Lee PW, Nordlee JA, Koppelman SJ, Baumert JL and Taylor SL, Measuring parvalbumin levels in fish muscle tissue: relevance of muscle locations and storage conditions. Food Chem 135:502-507 (2012).

45. Ruethers $T$, Taki AC, Nugraha R, Karnaneedi S, Cao TT, Dai D, Shanmuganathan T, Leeming $M$, Nie S, Williamson NA, Mehr SS, Campbell DE and Lopata AL, Abstracts from the European Academy of Allergy and Clinical Immunology Congress, 01-05 June 2019, Lisbon, Portugal. Allergy 74 Suppl 106:3915 (2019).

46. Kalic T, Morel-Codreanu F, Radauer C, Ruethers T, Taki AC, Swoboda I, Hilger C, HoffmannSommergruber K, Ollert M, Hafner C, Lopata AL, Morisset M, Breiteneder H and Kuehn A, Patients Allergic to Fish Tolerate Ray Based on the Low Allergenicity of Its Parvalbumin. J Allergy Clin Immunol Pract 7:500-508 e511 (2019).

47. Stephen JN, Sharp MF, Ruethers T, Taki A, Campbell DE and Lopata AL, Allergenicity of bony and cartilaginous fish - molecular and immunological properties. Clin Exp Allergy 47:300-312 (2017).

48. Yang RQ, Chen $Y L$, Chen F, Wang $H$, Zhang $Q$, Liu GM, Jin $T$ and Cao MJ, Purification, Characterization, and Crystal Structure of Parvalbumins, the Major Allergens in Mustelus griseus. $J$ Agric Food Chem 66:8150-8159 (2018).

49. Cai QF, Liu GM, Li T, Hara K, Wang XC, Su WJ and Cao MJ, Purification and characterization of parvalbumins, the major allergens in red stingray (Dasyatis akajei). J Agric Food Chem 58:12964-12969 (2010).

50. Dulvy NK, Simpfendorfer CA, Davidson LNK, Fordham SV, Brautigam A, Sant G and Welch DJ, Challenges and Priorities in Shark and Ray Conservation. Curr Biol 27:R565-R572 (2017).

51. Lam VYY and de Mitcheson YS, The sharks of South East Asia - unknown, unmonitored and unmanaged. Fish and Fisheries 12:51-74 (2011). 
52. Dent $\mathrm{F}$ and Clarke $\mathrm{S}$, State of the global market for shark products. FAO Fisheries and Aquaculture technical paper:I (2015).

This article is protected by copyright. All rights reserved. 


\section{TABLES and FIGURES (captions)}

Table 1. Details of investigated species, including common and scientific name as well as taxonomic classification.

Note: All fish species, except Atlantic cod, are consumed in the Asia-Pacific region and most are traded globally. Fish species with a cross ${ }^{\dagger}$ are native to Europe/North America. R= Raw and $\mathrm{H}=$ Heated extracts. The grey shaded boxes visualize that the specific kit detected the corresponding extract, while the white box marks no detection.

Figure 1. ELISA kit specific standard curves. The curves correspond to the quality certificates provided by the manufacturer. The blue full triangle and red half triangle represent the experimental result of the raw and heated extracts from Atlantic cod (Gadus morhua), respectively. A shows the standard curve and results for AgraQuant Fish ELISA kit (Romer Labs, Austria), B for the Common Bone Fish Antigen EIA ELISA kit, version 2 (XEMA, Russia), and C for the Common Bone Fish Antigen EIA ELISA kit, version 3 (XEMA, Russia).

Figure 2. Detection of bony fish. Results are shown as bar graphs of the raw (solid blue) and heated (pattern red) bony fish extracts for A ELISA kit 1, B ELISA kit 2, C ELISA kit 3. The numbers on the x-axis refer to species grouped according to the taxonomic order presented in table 1. For the respective ELISA kit the LOQ (equals Standard 1) is shown as solid pink, $1 \frac{1}{2}$ LOQ (LOD) as solid purple line. The result for the reference heated cod extracts 
is shown as dotted red line and for the reference raw cod extract is shown as blue dotted line.

Figure 3. Detection of canned fish. Results are shown as bar graphs of the canned fish samples. Details of samples salmon 1-8, tuna 1-7, and pilchard/sardine 1-2 are presented in Table A.1. The LOQ (equals Standard 1) is shown as solid pink line, $1 / 2$ LOQ (LOD) as solid purple line. The result for the reference heated cod extracts as dotted red line for the respective ELISA kits.

Figure 4. Detection of non-bony fish vertebrates and shellfish. Results are shown as bar graphs of raw (solid fill) and heated (pattern fill) extracts from cartilaginous fish, non-fish vertebrates and shellfish for A ELISA kit 1, B ELISA kit 2, C ELISA kit 3. The numbers on the $x$-axis refer to species grouped according to the taxonomic order presented in table 1 . For the respective ELISA kit the LOQ (equals Standard 1 ) is shown as solid pink and $1 / 2$ LOQ (LOD) as solid purple line.

\section{Appendices - SUPPORTING INFORMATION}

\section{Table S1: Details of analyzed canned fish.}

Note: The grey shaded boxes visualize that the specific kit detected the corresponding extract, while the white box marks no detection.

Figure S1. Taxonomic relationship of investigated species. 
Figure S2. Protein profile of cod extracts used as references and controls. A shows the SDS-PAGE profile of raw (RE) and heated (HE) extracts from Atlantic cod (Gadus morhua). B and C show corresponding immunoblots using a parvalbumin- and tropomyosinspecific antibody, respectively. 
Table 1. Details of investigated species, including common and scientific name as well as taxonomic classification.

\begin{tabular}{|c|c|c|c|c|c|c|c|c|c|c|c|c|}
\hline & \multirow[b]{3}{*}{ common name(s) } & \multicolumn{6}{|c|}{ detection by } & \multirow[b]{3}{*}{ scientific name } & \multirow{2}{*}{\multicolumn{4}{|c|}{ taxonomic classification }} \\
\hline & & kit & & kit 2 & & kit & & & & & & \\
\hline & & $\mathrm{R}$ & $\mathrm{H}$ & $\mathrm{R}$ & $\mathrm{H}$ & $\mathrm{R}$ & $\mathrm{H}$ & & family & order & class & phylum \\
\hline 1 & Asian swamp eel & & & & & & & Monopterus albus & Synbranchidae & Anguilliformes & \multirow{19}{*}{ Actinopterygii } & \multirow{19}{*}{ Chordata } \\
\hline 2 & splendid alfonsino & & & & & & & Beryx splendens & Berycidae & \multirow[b]{2}{*}{ Beryciformes } & & \\
\hline 3 & orange roughy ${ }^{\dagger}$ & & & & & & & $\begin{array}{l}\text { Hoplostethus } \\
\text { atlanticus }\end{array}$ & Trachichthyidae & & & \\
\hline 4 & $\begin{array}{l}\text { European } \\
\text { pilchard }^{\dagger}\end{array}$ & & & & & & & Sardina pilchardus & Clupeidae & Clupeiformes & & \\
\hline 5 & European carp $^{\dagger}$ & & & & & & & Cyprinus carpio & Cyprinidae & Cypriniformes & & \\
\hline 6 & Pacific cod & & & & & & & Gadus macrocephalus & \multirow{3}{*}{ Gadidae } & \multirow{4}{*}{ Gadiformes } & & \\
\hline 7 & Atlantic $\operatorname{cod}^{\dagger}$ & & & & & & & Gadus morhua & & & & \\
\hline 8 & $\begin{array}{l}\text { Southern blue } \\
\text { whiting }\end{array}$ & & & & & & & $\begin{array}{l}\text { Micromesistius } \\
\text { australis }\end{array}$ & & & & \\
\hline 9 & blue grenadier & & & & & & & $\begin{array}{l}\text { Macruronus } \\
\text { novaezelandiae }\end{array}$ & Merlucciidae & & & \\
\hline 10 & milkfish & & & & & & & Chanos chanos & Chanidae & Gonorynchiformes & & \\
\hline 11 & $\begin{array}{l}\text { flathead grey } \\
\text { mullet }\end{array}$ & & & & & & & Mugil cephalus & Mugilidae & Mugiliformes & & \\
\hline 12 & ling/pink cusk-eel & & & & & & & Genypterus blacodes & Ophidiidae & Ophidiiformes & & \\
\hline 13 & Chinese icefish & & & & & & & Neosalanx tangkahkeii & Salangidae & Osmeriformes & & \\
\hline 14 & Nile tilapia & & & & & & & Oreochromis niloticus & Cichlidae & \multirow{6}{*}{ Perciformes } & & \\
\hline 15 & $\begin{array}{l}\text { king George } \\
\text { whiting }\end{array}$ & & & & & & & Sillaginodes punctatus & \multirow[t]{2}{*}{ Sillaginidae } & & & \\
\hline 16 & sand whiting & & & & & & & Sillago ciliata & & & & \\
\hline 17 & $\begin{array}{l}\text { yellowtail } \\
\text { kingfish/amberjack }\end{array}$ & & & & & & & Seriola lalandi & Carangidae & & & \\
\hline 18 & $\begin{array}{l}\text { blue eye trevalla/ } \\
\text { bluenose warehou }\end{array}$ & & & & & & & $\begin{array}{l}\text { Hyperoglyphe } \\
\text { antarctica }\end{array}$ & Centrolophidae & & & \\
\hline 19 & $\begin{array}{l}\text { mahi mahi/ } \\
\text { dolphinfish }\end{array}$ & & & & & & & Coryphaena hippurus & Coryphaenidae & & & \\
\hline
\end{tabular}




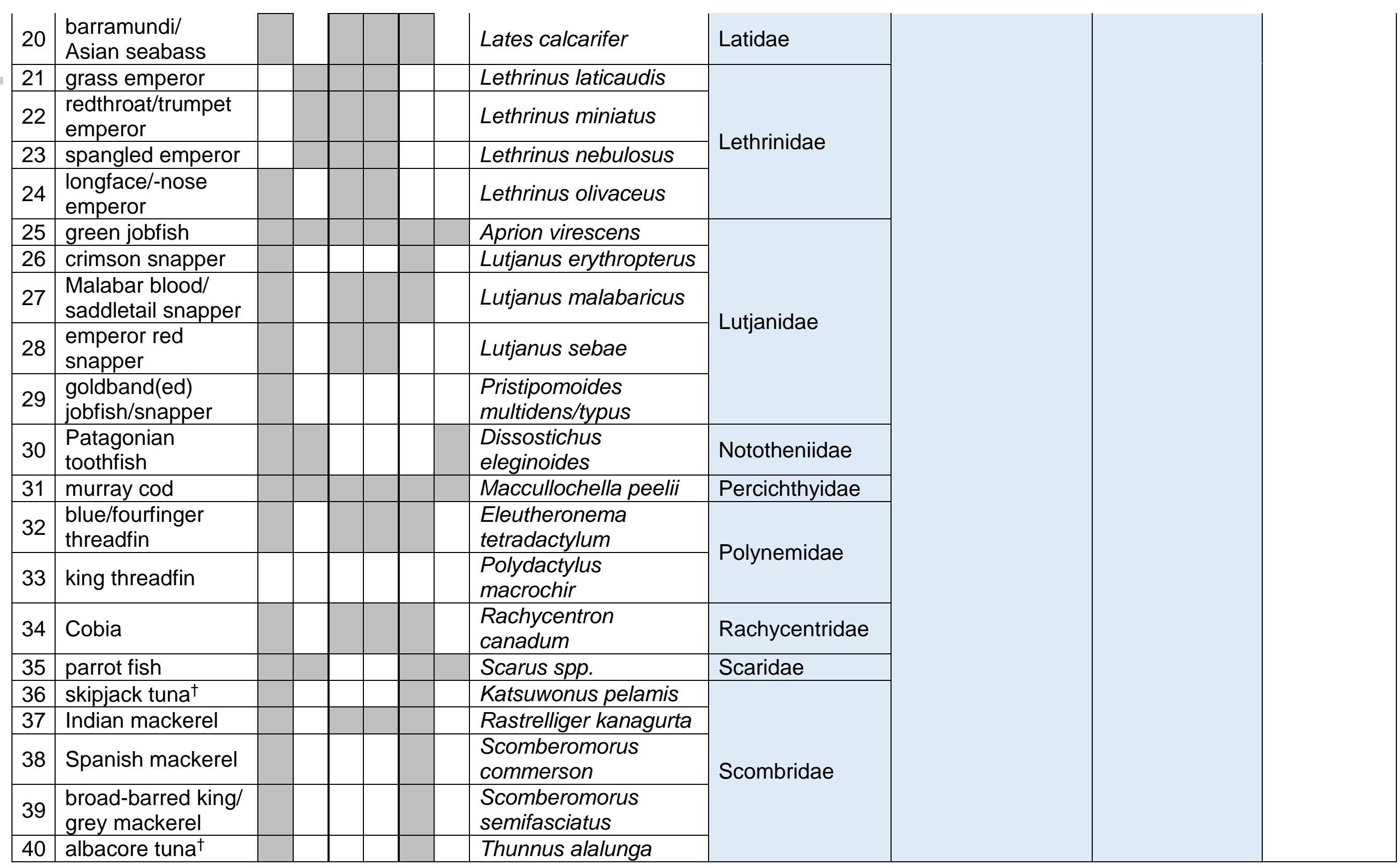




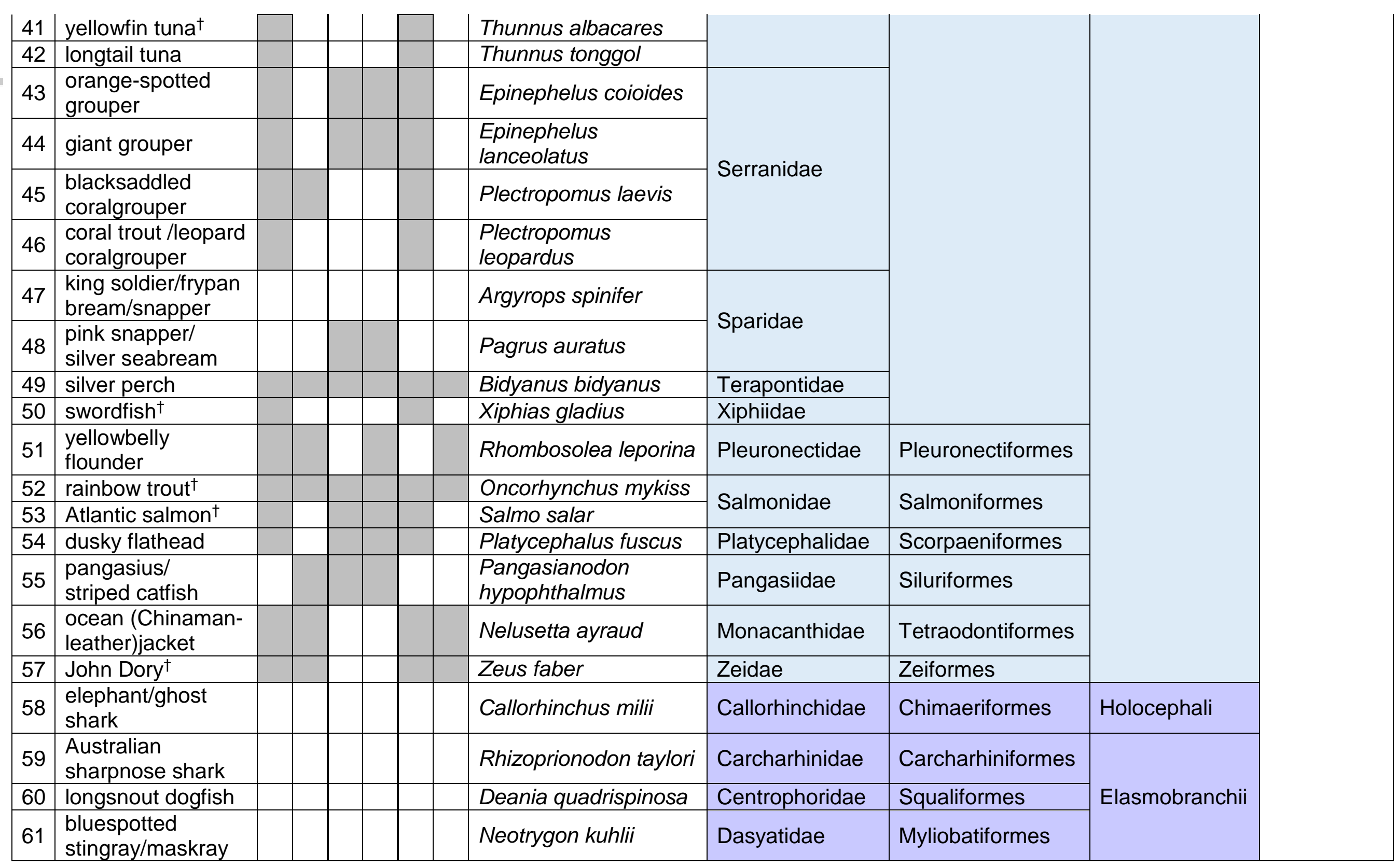




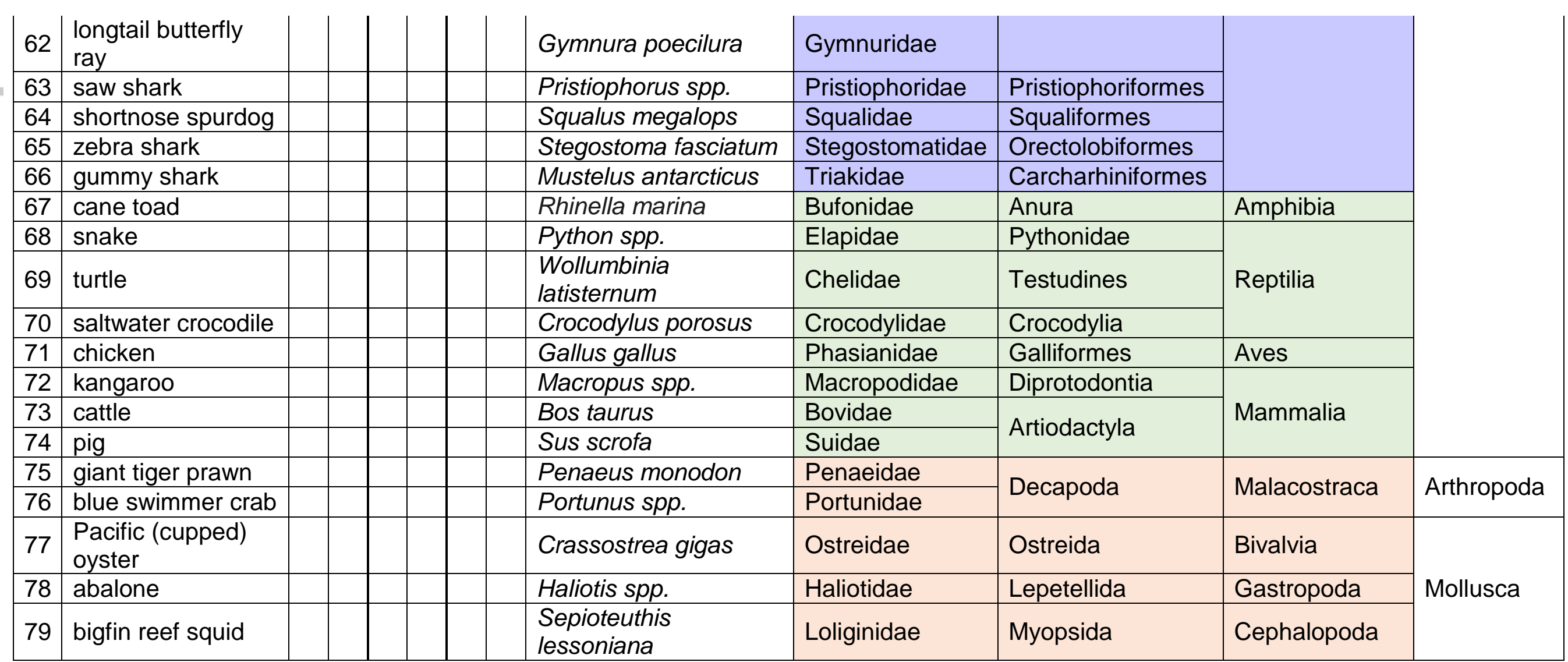

Note: All fish species, except Atlantic cod, are consumed in the Asia-Pacific region and most are traded globally. Fish species with a cross ${ }^{\dagger}$ are native to Europe/North America. $\mathrm{R}=\mathrm{Raw}$ and $\mathrm{H}=$ Heated extracts. The grey shaded boxes visualize that the specific kit detected the corresponding extract, while the white box marks no detection. 


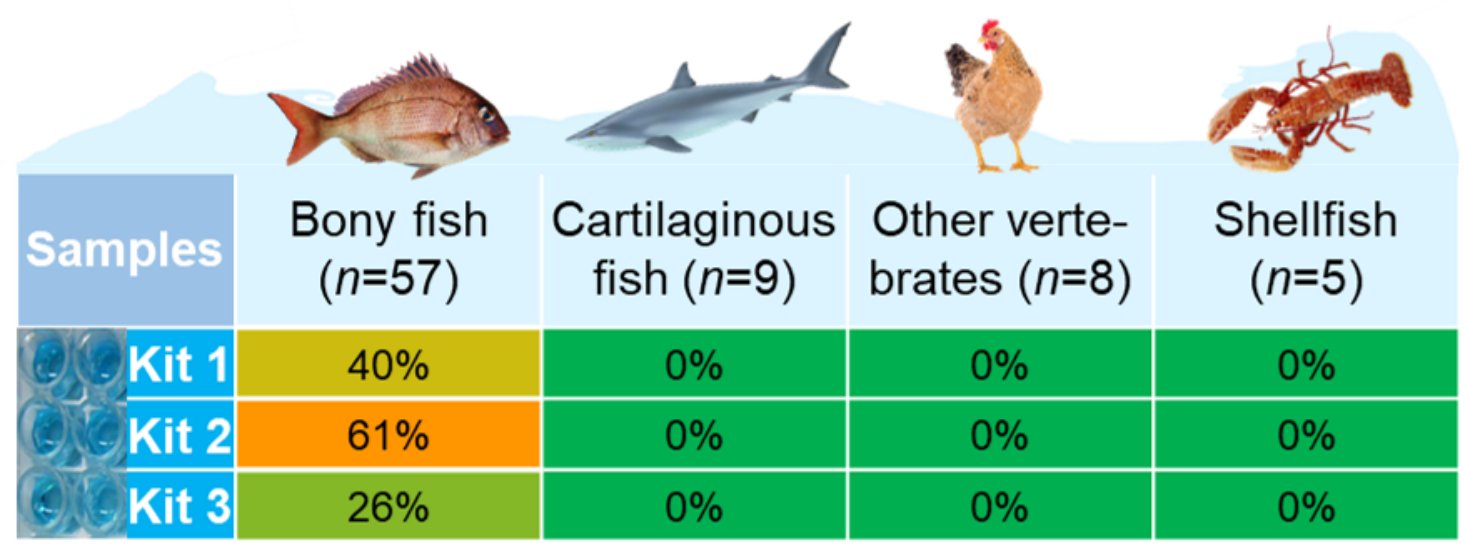

JSFA_10451_Cover Submission_fish ELISA_Feb20.tif 

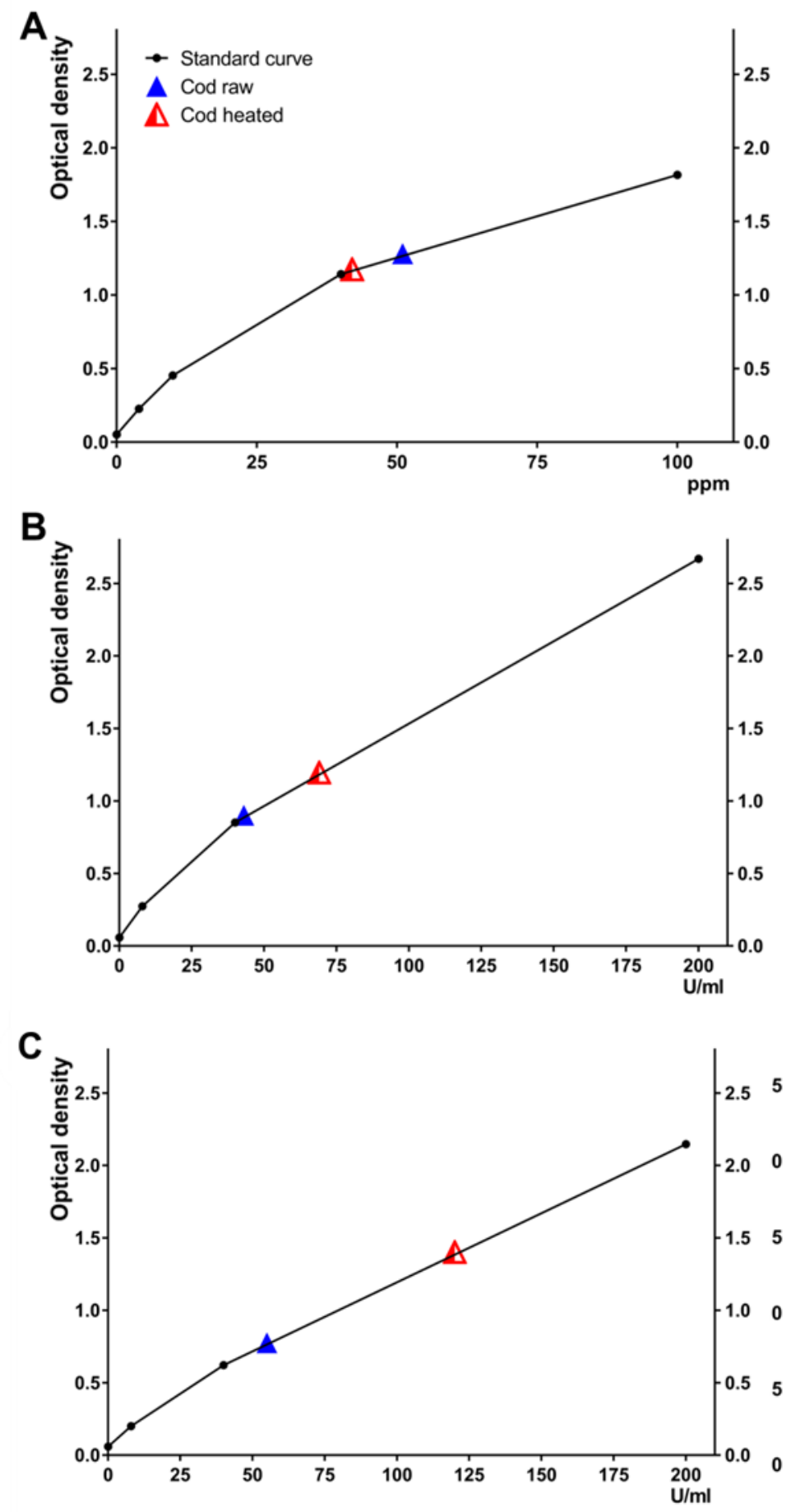

JSFA_10451_Fig 1_Ruethers_fish ELISA_Feb20.tif

This article is protected by copyright. All rights reserved. 

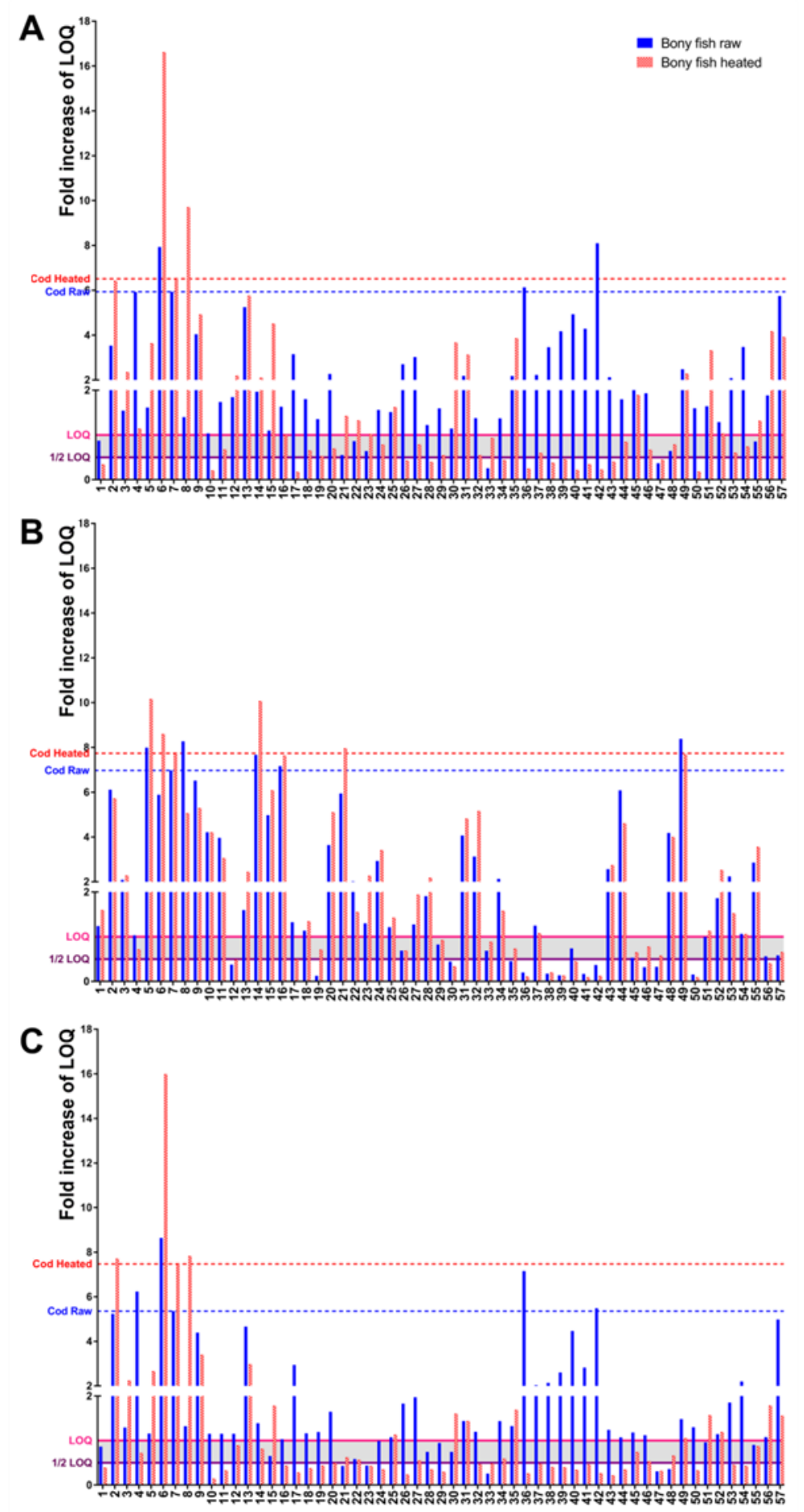

JSFA_10451_Fig 2_Ruethers_fish ELISA_Feb20.tif 


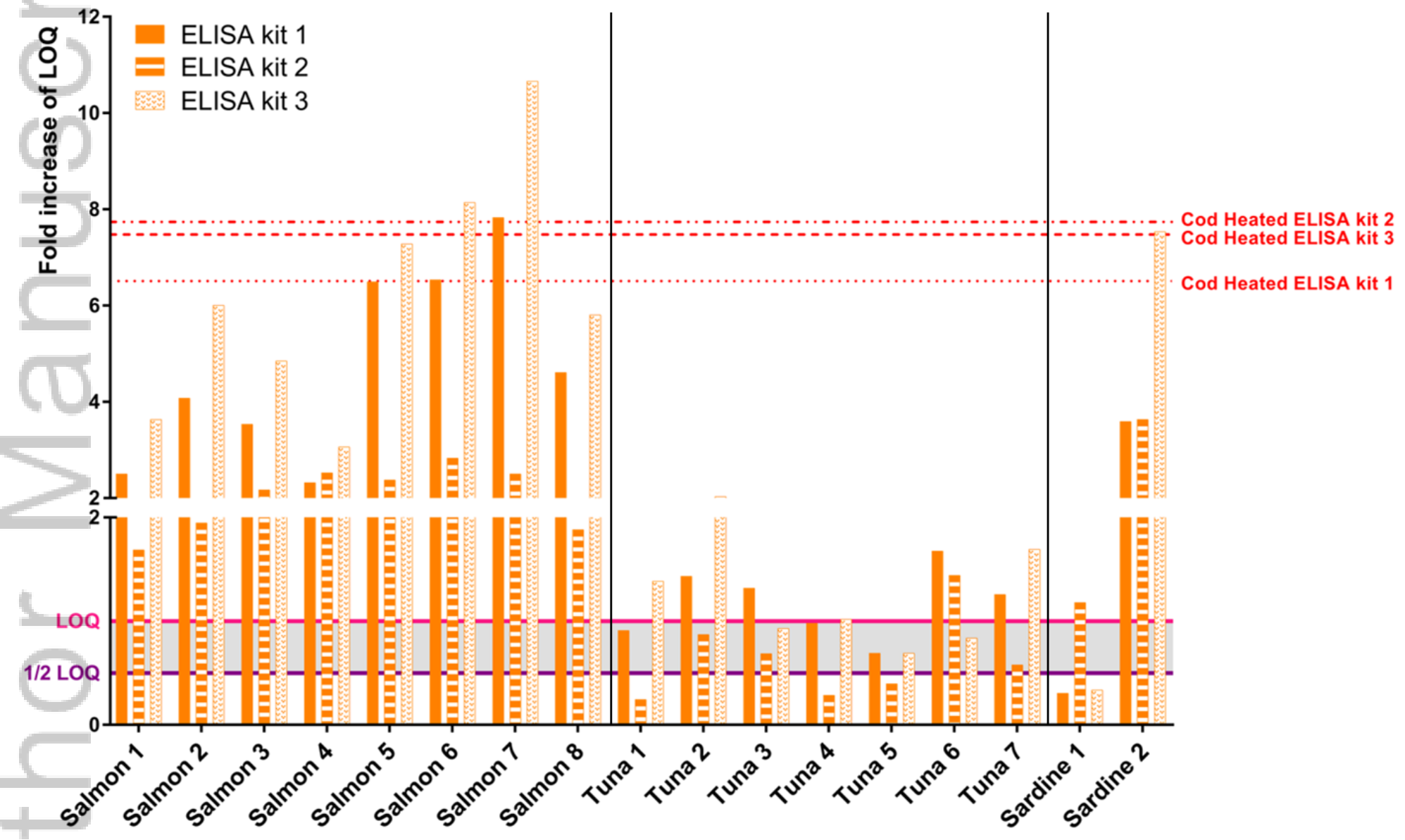

JSFA_10451_Fig 3_Ruethers_fish ELISA_Feb20.tif 

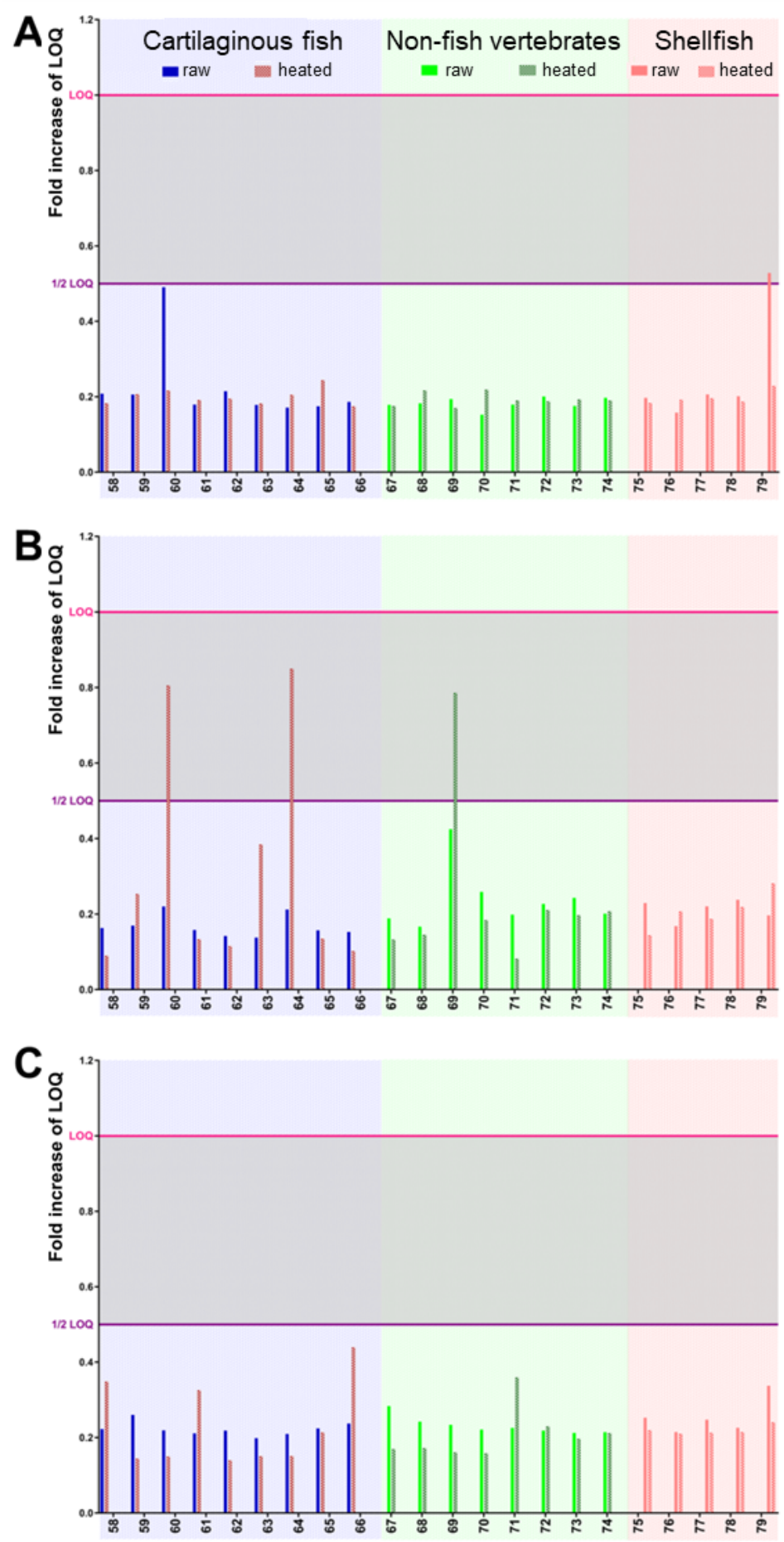

JSFA_10451_Fig 4_Ruethers_fish ELISA_Feb20.tif

This article is protected by copyright. All rights reserved. 


\section{University Library}

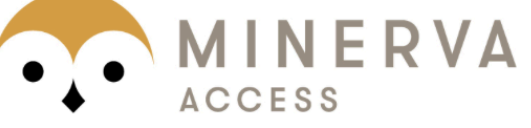

A gateway to Melbourne's research publications

Minerva Access is the Institutional Repository of The University of Melbourne

Author/s:

Ruethers, T;Taki, AC;Khangurha, J;Roberts, J;Buddhadasa, S;Clarke, D;Hedges,

CE;Campbell, DE;Kamath, SD;Lopata, AL;Koeberl, M

Title:

Commercial fishELISAkits have a limited capacity to detect different fish species and their products

Date:

2020-06-15

Citation:

Ruethers, T., Taki, A. C., Khangurha, J., Roberts, J., Buddhadasa, S., Clarke, D., Hedges, C. E., Campbell, D. E., Kamath, S. D., Lopata, A. L. \& Koeberl, M. (2020). Commercial fishELISAkits have a limited capacity to detect different fish species and their products. JOURNAL OF THE SCIENCE OF FOOD AND AGRICULTURE, 100 (12), pp.4353-4363. https://doi.org/10.1002/jsfa.10451.

Persistent Link:

http://hdl.handle.net/11343/275910 